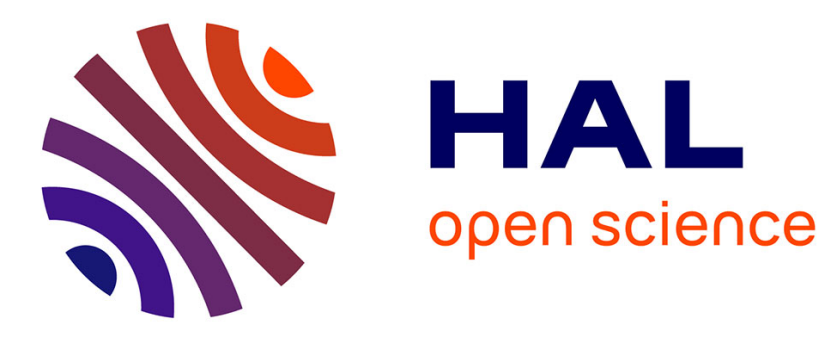

\title{
Supporting Mobile Connectivity: from Learning Scenarios to Multichannel Devices
}

José Rouillard, Yvan Peter, Jean-Claude Tarby, Thomas Vantroys, Vincent Chevrin

\section{- To cite this version:}

José Rouillard, Yvan Peter, Jean-Claude Tarby, Thomas Vantroys, Vincent Chevrin. Supporting Mobile Connectivity: from Learning Scenarios to Multichannel Devices: Special Issue on "Learning as a Ubiquitous and Continuous Communication Attitude". International Journal of Continuing Engineering Education and Life-Long Learning (IJCEELL), 2008, 18 (4), pp.396-410. hal-00825960

\section{HAL Id: hal-00825960 \\ https://hal.science/hal-00825960}

Submitted on 24 May 2013

HAL is a multi-disciplinary open access archive for the deposit and dissemination of scientific research documents, whether they are published or not. The documents may come from teaching and research institutions in France or abroad, or from public or private research centers.
L'archive ouverte pluridisciplinaire HAL, est destinée au dépôt et à la diffusion de documents scientifiques de niveau recherche, publiés ou non, émanant des établissements d'enseignement et de recherche français ou étrangers, des laboratoires publics ou privés. 


\title{
Supporting mobile connectivity: from learning scenarios to multi-channel devices
}

\author{
José Rouillard*, Yvan Peter, \\ Jean-Claude Tarby, \\ Thomas Vantroys \\ and Vincent Chevrin
}

LIFL, Université des Sciences et Technologies de Lille, 59655 Villeneuve d'Ascq cedex, France E-mail: jose.rouillard@univ-lille1.fr E-mail: yvan.peter@univ-lille1.fr E-mail: jean-claude.tarby@univ-lille1.fr E-mail: thomas.vantroys@univ-lille1.fr E-mail: vincent.chevrin@univ-lille1.fr *Corresponding author

\begin{abstract}
The introduction of distance learning does not only bring a wider audience, but also much more diversity among the learners: first, because it can be integrated more easily into a Life-long Learning strategy; secondly, because the learners are not restricted to a single area and thus learners from different countries and with different cultures follow the curriculum. We have observed this in various DL diplomas in which we participate. In this article, we will shed some light on the difficulties and challenges arising from these multicultural settings. Based on our research work, we would like to insist on two particular points which are the necessity to adapt the pedagogical settings (e.g. pedagogical scenarios) according to the learners' behaviour to overcome unforeseen problems due to cultural differences and the importance of considering mobile technologies to overcome limited access to the technology in developing countries and to ensure continuous interaction among learners and with tutors.
\end{abstract}

Keywords: Distance Learning; DL; multi-modal and multi-channel communication; pedagogical scenario; workflow.

Reference to this paper should be made as follows: Rouillard, J., Peter, Y., Tarby, J-C., Vantroys, T. and Chevrin, V. (2008) 'Supporting mobile connectivity: from learning scenarios to multi-channel devices', Int. J. Continuing Engineering Education and Life-Long Learning, Vol. 18, No. 4, pp.395-409.

Biographical notes: José Rouillard is an Associate Professor in Computer Science at the University of Lille 1. He obtained his PhD in 2000 from the University of Grenoble (France) in the field of Human-Computer Interfaces. $\mathrm{He}$ is interested in HCI plasticity, multi-modality and multi-channel interfaces. He has written more than 60 papers and is now engaged in research on mobility and pervasive/ubiquitous computing.

Copyright (C) 2008 Inderscience Enterprises Ltd. 
Yvan Peter is an Associate Professor in Computer Science at the University of Lille 1. He obtained his PhD in 1998 from the University of Franche-Comté, France in the field of Middleware Engineering. He is interested in the development of flexible software to support learning and collaboration. He is a member of the scientific committee of the European Network of Excellence Kaleidoscope in the field of Technology Enhanced Learning and responsible for the national project $\mathrm{p}$-LearNet about pervasive learning environments.

Jean-Claude Tarby received his $\mathrm{PhD}$ in Computer Science from the University of Toulouse I in 1993. Since 1995, he is an Assistant Professor at the LIFL Laboratory of University Lille 1. His research is focused on the modelling and the design of cooperative applications based on user's tasks. His interests include multimodality, meta-modeling, user interface design methods, groupware and traces of activities.

Thomas Vantroys is an Associate Professor in Computer Science at the University of Lille 1, France. He obtained his $\mathrm{PhD}$ in 2003 from the University of Lille 1 in the field of flexible workflow engine for e-learning. $\mathrm{He}$ is interested in the SOA and more particularly in service orchestration in the context of pervasive-learning.

Vincent Chevrin is Computer Science PhD from the University of Lille in France. His PhD has largely focused on multi channels aspects in the context of communications and transactions between organisations and users in the eCommerce area. Currently, he is an Engineer of application and works in the field of marketing. He also continues to work on some challenges close to the multi-channel and pervasive applications issues.

\section{Introduction}

For a number of curriculum, Distance Learning (DL) has brought not only a wider audience, but also much more diversity among the learners. This is due to two reasons. First, DL can be integrated more easily into a Life-long Learning (LFL) strategy bringing experimented people to assist to the courses. For this reason, the curriculum is attended by learners which have a more diverse experience.

Secondly, the learners are not restricted to a local area where they attend the courses and thus learners from different countries and with different cultures follow the curriculum.

We have observed this in various DL diplomas in which we participate. The first one is a Master in Computer Science, including people from France and from French speaking countries (Tunisia, Algeria, etc.). The second diploma is a Master in Pedagogical Engineering.

In this article, we will shed some light on the difficulties and challenges arising from these multi-cultural settings. We would like to insist on two particular points which are the necessity to adapt the pedagogical settings (e.g. pedagogical scenarios) according to the learners' behaviour to overcome unforeseen problems due to cultural differences and the importance of considering mobile technologies to overcome limited access to the technology in developing countries and to ensure continuous interaction among learners and with tutors. 
Based on the illustration of these two points drawn from our practical experience, we describe some research work that we have done towards providing flexibility of the pedagogy and of the platform access modes. On the former, we present an approach based on the modelling of pedagogical scenario that illustrate the tutors' and learners' activities and a solution for the enactment and revision of these scenarios at runtime.

On the latter, we give an overview of the potential of multi-channel communication to provide ubiquitous access to the learning management platforms. To take into account a large range of potential access devices, we need to support different kinds of interaction, from simple modes based on widespread devices, such as mobile phone, for instance to very rich modes (several devices and channels at the same time).

To conclude we link these different solutions in a coherent manner to provide a comprehensive and flexible solution to support the kind of flexibility needed to manage the diversity provided by the cultural and technical differences among learners.

\section{Practices and issues of transnational DL}

Since more than 30 years, our Institute has a long experience in DL and E-Learning (EL) with a much diversified population composed of people who are workless and need to have a reorientation, and people who look for diploma. For the former, we practice DL by 'classical' ways such as fax, phone, postal mail, etc. For the latter, we opened two professional masters in EL, one in 1998 in computer science, and one in 2003 in computer science and learning science. These EL diplomas are open for French-speaking people. Until now, the students were from Tunisia, Rwanda, Syria and obviously France.

After several years of experience in these two EL diplomas, and beside the classical problems for EL like the lack of motivation mainly due to technical problems and isolation, we have noticed some particular points which are relevant to the multi-cultural dimensions. These dimensions may be factorised into five categories which are now detailed in the remainder of this part 2.

\subsection{Academically-Based Education vs. Life-long Learning}

Our two masters are composed of persons coming from the academic world (Academically-Based Education; ABE) and from the business world (LFL). For the moment, ABE people are less concerned by EL form although the demand for ABE could be more important in the next years because of the evolution of teaching in the French University. Consequently, the LFL people are the most concerned by our EL diploma.

Since our diplomas must be the same in presence and in EL (to comply with legal obligations), we had to adapt our teaching to these two very different populations. This adaptation is based onto five criteria:

- Age. The ABE people are about 23 years old, but the LFL people are about 40.

Therefore, we cannot talk to the LFL people in the same manner as to the ABE people. Often LFL are older than their teachers and tutors.

- Culture. The centres of interest are very different between the ABE and LFL people. In the same way, the ABE students have not the same experience about (professional) life as the LFL learners. 
- Motivation. Even if both want to pass their degree their, motivation is very different. The ABE students want a diploma to obtain a work when the LFL learners want to evolve in their company, to have their work officially recognised by their boss, or to move to another work or company. Therefore, they do not ask the same type of teaching and competencies.

- Way of life. The LFL learners are usually married and have children opposite to the $\mathrm{ABE}$ students who are most often single. When we ask to the ABE students to work during $8-12$ hours per day, we cannot do it for the LFL learners, because they must manage their family life.

- Work style. An ABE student is available during all the week, but an LFL learner is not. Depending on their work and their responsibilities, the LFL students may work on their lessons and homework every day (i.e. one hour per day), once per week (during the week end for instance), etc. More, some LFL people can study at work, but others cannot. Some of them prefer to work off-line (week-end in the country), others on-line. Some of them want only the PDF documents, whereas others request multimedia documents.

For all of these differences, we have to be able to adapt our teachings to satisfy the two-learner populations. For the moment, these adaptations are made ad hoc depending upon the people, the diploma, etc.

\section{Local and distance students}

\subsection{Problems about time lag}

Some of our LFL learners are living in France, some others are living close to France, and others are living very far from France. These distances can be very harmful for real time examinations when the time lag is too high (until seven hours at the moment). That means that we either must avoid to do real time examinations or to plan such examination with regard to the time lags. The first solution implies a modification of teaching, the second solution implies to look after the duration of examinations and the start and finish time; we cannot ask to a student to have an examination at $3.00 \mathrm{am}$ or at $11.00 \mathrm{pm}$ !

\subsection{Problems about cultural differences}

At the beginning, we had some difficulties to write examination subjects comprehensible by everybody. This problem was due to cultural differences. Actually you cannot write subject which are very specific to your country or countries around you. For instance, people from Africa may have some difficulties to understand an examination about ski station, and people from Peru may not know what Société Nationale des Chemins de Fer is (which is the national rail company in France). The cultural difference must be identified at the beginning and the teaching must be adapted consequently. 


\subsection{Problems about life way}

We encountered some unexpected difficulties because some countries have political or economical problems.

Concerning economical limitations, we had for instance a student in Rwanda who had to drive $100 \mathrm{~km}$ each time that he wanted to be connected to our EL platform (he had to go to the next big town to find a 'cyber coffee'). Consequently, he was unable to follow the teaching in the same manner than people who are permanently online. On top of that, the work of this student was liable to water... Why? Because when the rains were not sufficient, there was not enough water in barrages and so electricity in towns and villages! One solution to this problem of lack of water could be the $\$ 100 \mathrm{PC}$ (cf. Figure 1). This solution is very interesting to be independent of electricity. More of that, this new computer should imply deep thinking for teachers about a 'new EL for this material...' Another solution is to adapt our teaching to other channels (for instance SMS, phone, etc.).

Figure 1 The '\$100' PC (see online version for colours)

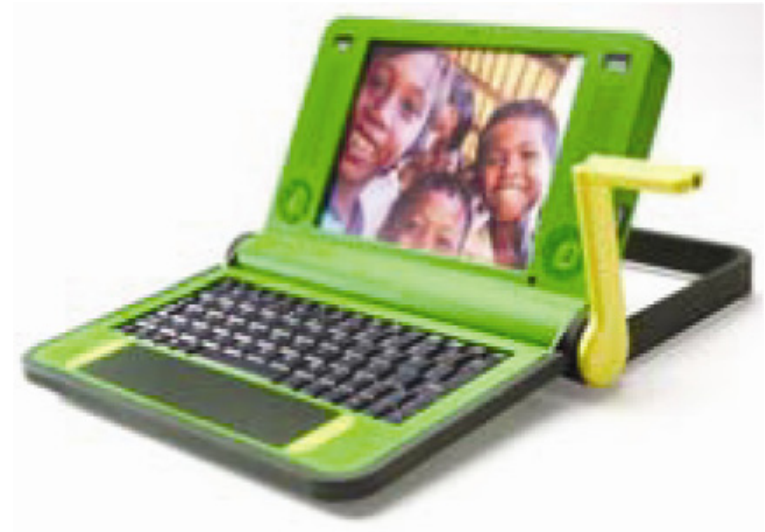

Source: http://laptop.media.mit.edu.

Concerning political limitations, Syria decided to block lots of internet connections. The students can send e-mails, but they were unable to surf on all the websites that we provided, neither to upload files onto our platform. We had to find urgently some solutions to resolve these problems, CDROM sent by postal mail for instance.

\section{Supporting flexible pedagogical settings}

As Fischer (2001) pointed out, the challenge about pedagogical flexibility is not only to make information available to people at any time, at any place, and in any form, but also specifically to say the right thing at the right time in the right way. 
From the experience presented before, we can see that we need adaptation of the pedagogical setting. For this, we can have two approaches. First, at design time we can abstract from the concrete setting so as to keep the pedagogical objectives in mind. We can then deploy the abstract setting onto a concrete one. The second approach is more concerned with runtime issues and relies on the platform capability to support runtime adaptation of the activities. These two approaches are presented hereafter.

\subsection{Modelling and deployment of the pedagogical scenarios}

In order to support multi-cultural scenarios, we have to place the model in the middle of our system. In fact, the model driven approach is gaining momentum. We can note that by the fact the Model Driven Architecture (MDA, 2006) promoted by the Object Management Group (OMG, 2006) aims to separate the model of a system from its implementation. The main reason is that a change in the technology field, like changing from Java to $\mathrm{CA}$, should not have consequences on the model because models are persistent and implementation are transient. The same approach can be conducted in Learning Management Systems (LMS). This step resting on successive transformations of models is particularly well adapted to take into account the multi-cultural aspects. It is also well integrated with an approach based on pedagogical scenarios. Indeed, teaching that it carried out in a traditional way or by means of an LMS is not summarised by a simple access to pedagogical resources but through a progress into various coordinated activities (Koper, 2001) (Dillenbourg, 2002). These platforms should not be limited any more to the management of modules and contents, but must support the possibility of really creating complete courses of formation, i.e. organising and accompanying the pedagogical learning paths between various modules. The definition and exchange of these courses requires languages called Educational Modelling Language (EML), around whose efforts of standardisation are in hand (Pernin, 2006). These approaches by scenarios are close to processes handled within the workflow systems (Lin et al., 2001; Vantroys, 2003). The transformation process that allows carrying out our teaching scenarios is presented in Figure 2. Initially, the teacher describes in an abstract way (i.e. non-comprehensible by a computer) the various activities which compose the curriculum as well as the pedagogical aims and the necessary knowledge in order to follow the lessons. That corresponds in the MDA terminology to a Computer Independent Model. In a second time, a teaching engineer transcribes this representation in a more formal version. In our case, we use for that the language IMS-LD (IMS, 2003). That corresponds in the MDA terminology to a Platform Independent Model (PIM). This model has the advantage of being independent of any execution system and of any context. Thus, it is reusable and adaptable according to specific contexts' of use. In a third time, the teacher and the teaching engineer define specific rules to take account of the multi-culturality, like, e.g. execution of the scenario starting from the server or downloadable on the client according to the possible problems related to the infrastructure network. These rules are used for the transformation from PIM to PIM (see Figure 2). In our case, we make a transformation from IMS-LD to XML Process Definition Language (XPDL) (Workflow Management Coalition, 2002). We particularly use the extensibility properties of XPDL to take account of the rules. The last stage consists in deploying the model towards an execution system. 
Figure 2 Model transformation process (see online version for colours)

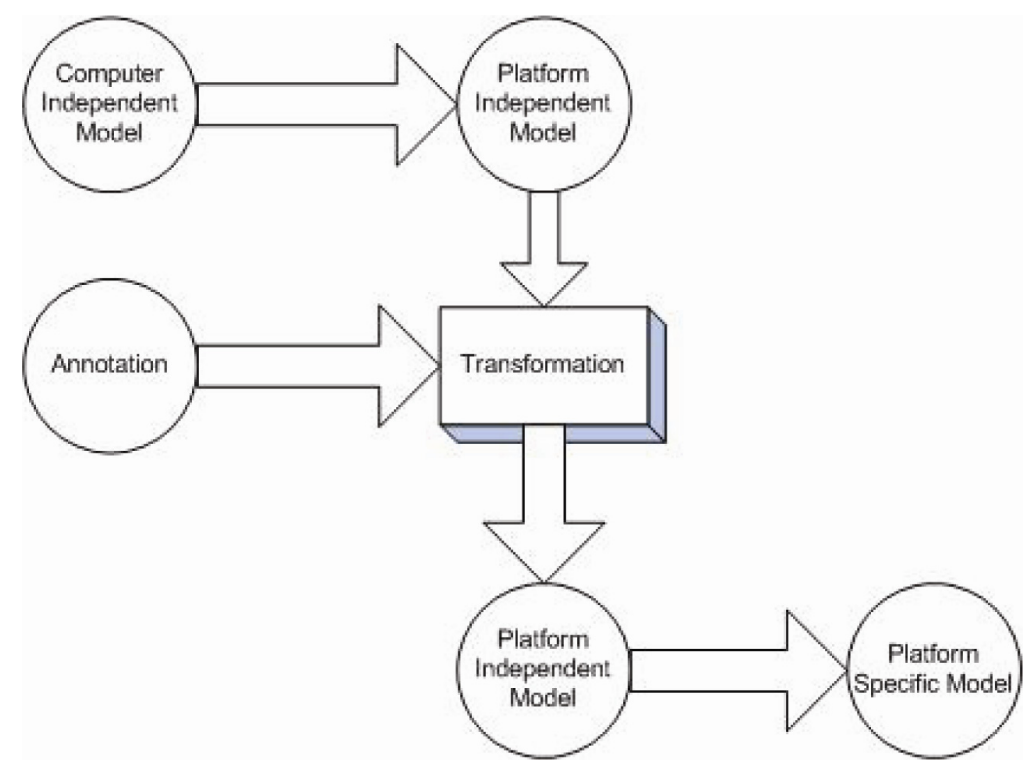

\subsection{Flexible support for the execution of pedagogical scenarios}

In our works, we focus at each step on the user (teacher, teaching engineer, learner, etc.) and we largely agree with Dourish (2004) when he says that

"[...] the focus of the design is not simply 'how can people get their work done,' but 'how can people create their own meanings and uses for the system in use'; and in turn, this suggests an open approach in which users are active participants in the emergence of ways of working."

The Cooperative Open Workflow (COW) platform (Peter and Vantroys, 2005), developed in our laboratory aims at producing a bridge between EML centred on the concept of activity and the systems of workflows in order to allow the execution of teaching scenarios. The three principal objectives are:

- to design a platform centred on the user (Bourguin and Derycke, 2005) where he/she acts throughout cycle of life to adapt and modify the system with the various contexts of use

- to relocate the models in the system kernel so they are constantly available and easy to handle

- to install mechanisms of flexibility allowing the realisation of the previous objectives.

This work is focused on a platform of teaching scenarios execution. We do not try to recreate a new LMS but rather to provide a new type of service which could be integrated in existing LMS. One of the keys of integration is to respect standards not only technically, but also pedagogically (use of standardised description languages). 
For technical interoperability, we chose a development based on standards J2EE particularly by supporting EJB. In order to extend integration in heterogeneous platforms, our system has a simple object access protocol interface in order to be integrated as a teaching scenarios management web service.

For the languages interoperability, we chose a meta-model approach and we weave bonds between the technical model and the model of the target teaching language. In the following, we will see the various properties which we implemented within our workflow engine in order to answer the identified needs concerning teachers and learners. The main characteristic relates to flexibility during the execution of the scenarios. Most of human activities are not predictable. Thus, it is utopian to believe that we can produce a platform meeting all the needs (present and future). Indeed, it is difficult for a teacher to express and formalise his/her scenario. It will often comprise fuzzy parts which will be cleared up during the execution. Another case can be that of students presenting learning difficulties. In this case, it will be necessary to modify the model in order to add for example additional modules. An alternative to the writing of the scenarios ex nihilo is to do reengineering from the execution logs in order to rebuild the used scenario and to formalise it by using for example the IMS-LD language (Barré, El-Kechaï and Choquet, 2005). It is then possible to replay this scenario via our platform. The flexibility brought by COW appears through two ways: the first relates to the possibility of modifying the scenario model during the execution, and the second relates to the possibility of modifying the interpretation of the scenario (i.e. the behaviour of the engine) on the fly.

The first axis is based on introspection and intercession mechanisms and also on the open implementation principle (Kiczales, des Rivières and Bobrow, 1991) which make possible to handle the model in order to add/delete/modify activities and to change paths between activities.

The second axis of flexibility relates to the execution engine itself. Indeed, it is impossible for the programmer to envisage all the use cases of the system. He can, however, use mechanisms of introspection and intercession to modify the behaviour of the platform and to adapt it to particular contexts of use. The way of managing the time constraints is a typical example of behaviour which one must be able to adapt. Indeed, the management of time is one of the other properties of COW. Taking into account of time makes it possible for example to limit the access to resources and tasks and avoids a too significant drift between fastest and slowest learners. The progress of the group is thus more homogeneous.

There are two temporal concepts: duration (minimal and/or maximum time) and dead lines. In order to manage them, we can use various strategies, such as for example taking into account the weekends or not. We can also dynamically adapt calculation according to contexts of use and users wishes. To allow various behaviours when constraints violation appears, we use the pattern strategy, for example, to send an e-mail to the student or the tutor, or to validate in an automatic way the activity.

One of the parameters to be taken into account is the individualisation of the process of training, i.e. the possibility for the students of a group to go at their own rhythm on certain sequences of the scenario. The teacher must specify at the modelling time the different kinds of sequences (individual or collective). A collective sequence does not mean that there is necessarily a collaborative task. Indeed, the teacher can just wish that all the students move ahead at the same time even for an individual work. To support these various sequences, we use the concept of sub-process, i.e. of subsets of scenario 
which will be activated only once during the execution for collective activities and once for each learner in the case of individual activities.

\section{Supporting mobile and ubiquitous learning}

In Democracy and Education, Dewey (1916) writes "Education is not an affair of 'telling' and being told, but an active and constructive process". For Dewey, education must be grounded in experience and active inquiry. Supporting mobile and ubiquitous learning is probably an important key of the problem.

Pervasive (or ubiquitous or ambient) learning relies on the 'always on' concept (see pervasive games). With an 'always on' education, available 24 hours a day, 7 days a week, anywhere, at any time, we are close to the notion of pervasive learning in a social aspect that connects learners not only to communities of people, but also devices and situations. Hence, learners can construct relevant and meaningful learning experiences, in locations and at times that they find relevant.

Pervasive learning is related to four key elements: community, relationality, autonomy and locationality. But as Thomas (2005) mentioned: "Pervasive learning is a field still in its infancy". This line of research goes by a number of names - ubiquitous computing (Weiser, 1991), context-aware computing (Dey, Abowd and Salber, 2001), pervasive computing (Ark and Selker, 1999), embodied interaction (Dourish, 2001), etc.

According to Bomsdorf (2005) (referencing Ogata, Yin and Yano, 2004), the main characteristics of ubiquitous learning are:

- Permanency. Learners can never lose their work unless it is purposefully deleted.

- Accessibility. Learners have access to their documents, data or videos from anywhere.

- Immediacy. Wherever learners are, they can get any information immediately.

- Interactivity. Learners can interact with experts, teachers, or peers in the form of synchronous or asynchronous communication.

- Adaptability. Learners can get the right information at the right place in the right way.

- Situating of instructional activities. The learning could be embedded in our daily life.

Supporting user mobility is for us to offer to learners the possibility to access the platform with mobile devices, for example. This access supposes document adaptation to the peripheral. Our aim is to propose a generic method to transform documents without making one document for each device.

As we address mobile device with few capacity (few memory, few persistent storage size), we wanted to have thin client. The most common used technology for thin client is at present time Hypertext Transfer Protocol (HTTP) and Hypertext Markup Language (HTML). With the concept of thin client, students can access their learning environment with any computer which has a web browser. However, learners use mobile systems as Personal Digital Assistant (PDA) and cellular phone. To support these devices, we build a platform based on a $\mathrm{N}$-tiers architecture (see Figure 3). 
Figure 3 Intermediation architecture for user access (see online version for colours)

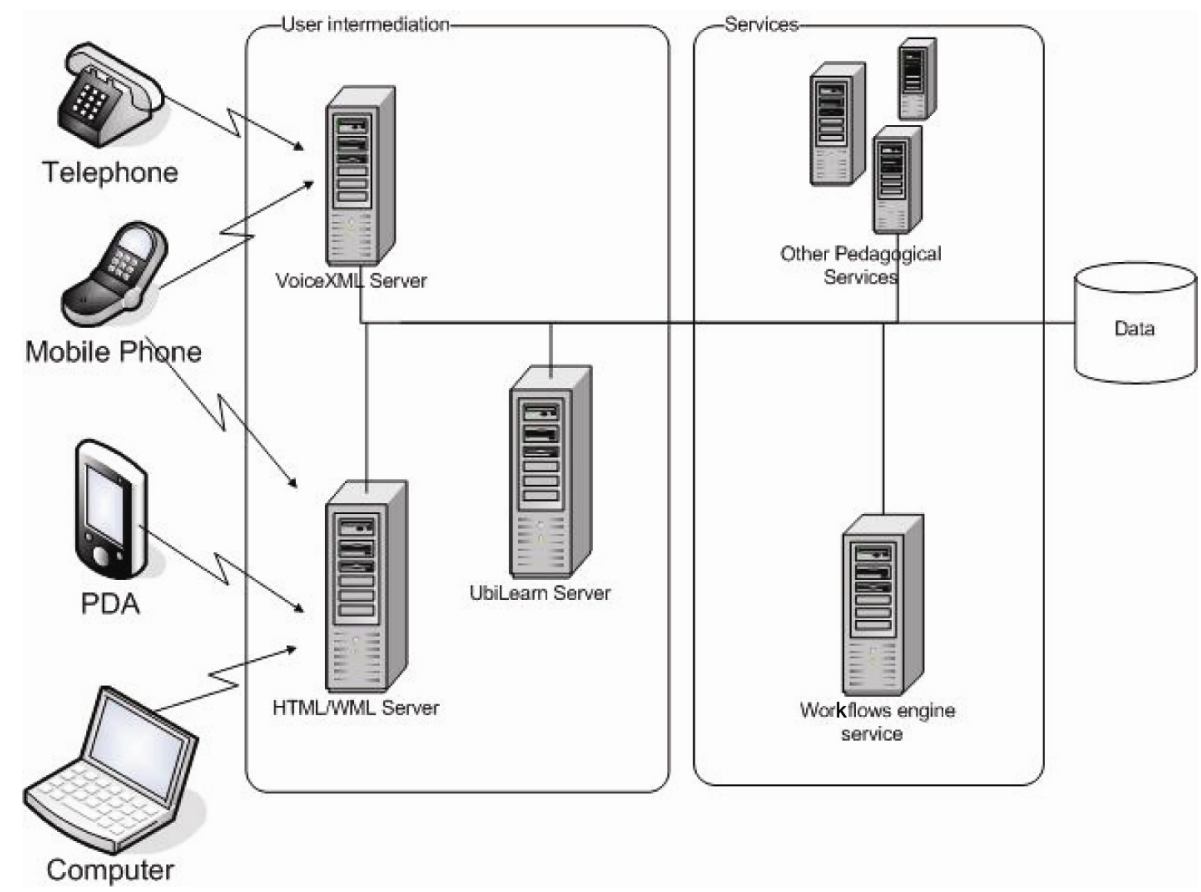

The first tier is composed by the devices used by learners to access the system. For the moment, we address the following devices:

- Personal Computer (PC/MAC). These devices offer the most interaction possibility (text, graphic, sound). As we wanted thin client, the student access the system with a web browser.

- $\quad P D A$. These devices have smaller screen, graphics must be resized. The student can access the system with a classical web browser or by using WAP (2006).

- Cellular phone. These devices offer two types of interactions. The first is the Wireless Application Protocol (WAP) which allows the visualisation of simple page web written in Wireless Markup Language (WML). The second interaction is the use of voice. For vocal interaction we use the VoiceXML language (VoiceXML, 2006) (Rouillard, 2004).

- Wired phone. The interactions are restricted to voice and telephone keypad. The student can access the system by calling a vocal server. The second tier is constituted of 'presentation' servers. These servers interact with the workflow server (third tier) to obtain the resources and apply the transformation to transmit document suitable to device used. The last tier is constituted of the database where resources and workflow state are stored. 


\subsection{Integration of any channels for seamless interaction between organisations and learners}

The possibility to use multiple channels when it is possible is really important in order to support mobile connectivity within learning scenarios. Moreover, this integration must take into account the issue of the rupture in the interaction, as for example, due to a network failure, etc.

For the moment, the only way of re-establishing the lost interaction depends on the learner's initiative of re-contacting the organisation. We sustain that this is a task that should be best done by the system itself, by detecting the rupture and calling automatically the leaner, using the best adapted channel taking into account the contexts. Here, there can be incompatibility between the context of the user (his/her profile, etc.) and the context of the organisation (from the pedagogical point of view, with the organisation rules, policies, etc.).

Figure 4 shows the conceptual view of our software architecture and the various kinds of context that we try to support and manage.

Figure 4 Conceptual view of our software architecture (see online version for colours)

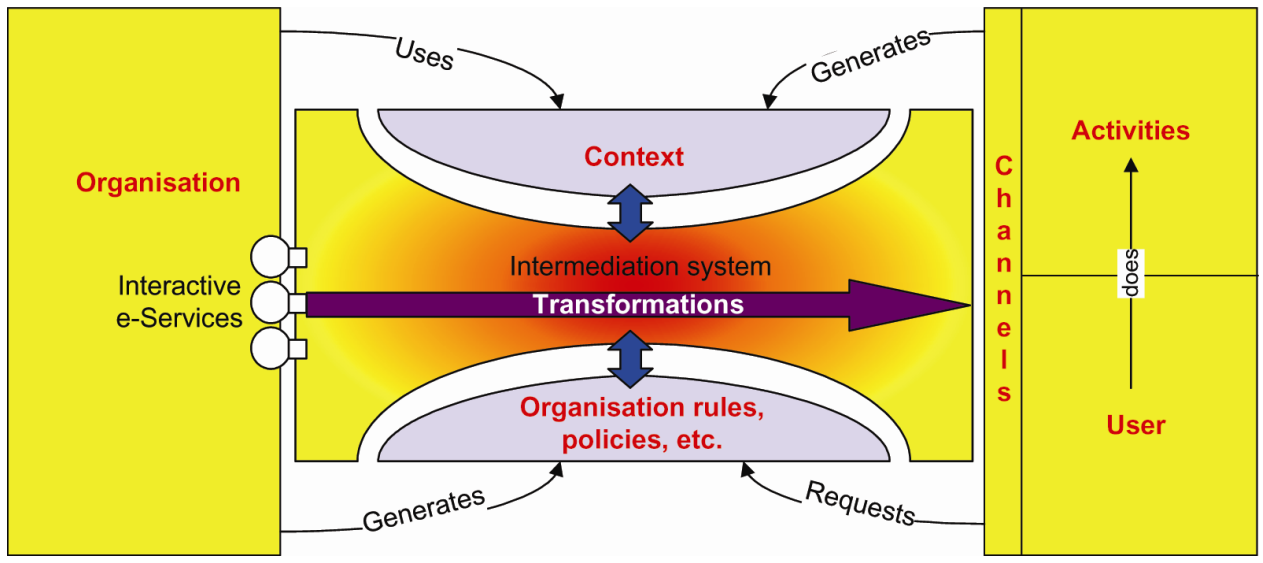

We can see in Figure 4 that we take account of two kinds of context:

- The 'policies' of the pedagogical organisation.

- The context provided (directly or not) by the user and used by the organisation in order to adapt the different services. In our prototype, we considered as a context, user preferences, channels in use, etc., but in a more ambitious perspective, we could have added geo-localisation, for example.

These two kinds of context are not always compatible. For example, if a learner has the habit of using services via her/his cellular phone, and the organisation does not deliver this service via this channel, the two contexts are not compatible. Therefore, our main interrogation is: how to manage these two contexts at the same time and in a coherent way? 
In order to illustrate the matter of contexts incompatibility, we present in Figure 5 a poetry example. The 'abstract pedagogical document' (an XML file) is transformed into a suitable document, according to the detected device, network capabilities, etc. Thus, this poetry will be readable not only on a traditional web browser (with in addition a picture of the author, for example), but also on a cellular phone using the WAP protocol.

Figure 5 The same pedagogical document presented through different channels (Web Browser and Wap browser) (see online version for colours)
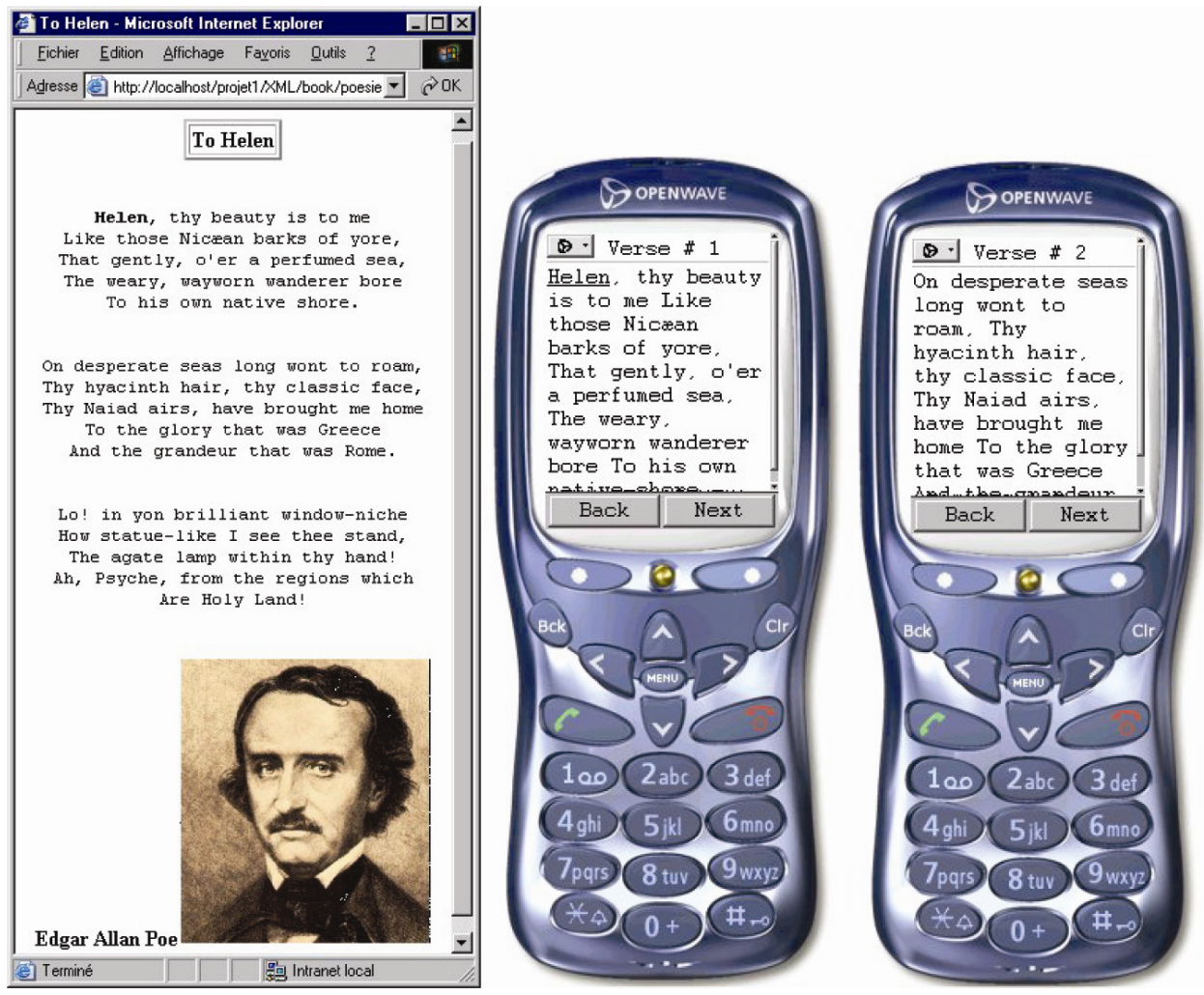

If the system cut the document technically (example 12 lines per screen) on the small phone screen, there is a risk to loose the pedagogical intention of the author that specially cut this poem with appropriate strophes. It will be exactly the same problem on an aural mode (VoiceXML language for example) if the Text-To-Speech (TTS) do not respect the punctuation and the pauses determined by the author.

\subsection{The inter-dependence between service composition and channels}

We will focus, now, more precisely on the nature of the dependence of service composition in relation to the channels used during the interaction.

In Chevrin, Derycke and Rouillard (2006), we have shown several scenarios where the composition of different e-services is conditioned by the nature of the channel and their interaction styles (direct manipulation vs. speech dialogue). Indeed from our previous evaluation of first prototypes (Derycke et al., 2003; Chevrin, Derycke and 
Rouillard, 2005; Chevrin, Rouillard and Derycke, 2005), combining different interaction channels, we have discovered that the concurrent accesses to several e-services conducted to different interleaving of the e-services primitives, depending of the channel in use. For example, reading and choosing from a table of items using a voice-guided interaction is different from a web interaction. In the former case, we cannot force the user to remember all the made choices and on the latter case it is possible to see the whole table on the screen. Therefore, the fragmentation of the source document (e.g. a form) must be adapted to the channel characteristics and to the interaction elements (such as widgets, vocal forms, etc.).

Moreover, we agree in affirming that the learner should be able to choose the channel that she/he wants and to execute the service that she/he wants. Obviously, this is only possible if the service is available for the selected channel.

In the field of software engineering, with the Service-Oriented Architecture (SOA) and in the business field (Rust and Kannan, 2003), we can show that SOA is also a good framework to solve this issue. We introduced the Interactive e-Service (e-SI) concept in Chevrin, Rouillard and Derycke (2005). Therefore, our model-driven architecture builds, so as to say, a bridge between three domains:

- web-services (the e-SI side, we can see in Figure 4)

- learning rules (the policies, pedagogical rules, in Figure 4)

- human-computer interface, for context-aware aspect (see Figure 4).

\section{Conclusions}

In this article, we have presented the main problems that we have to face considering learning as cultural migration through mobile connectivity. Adaptation is needed not only between academically-based education and life-long learning, but also between local and distant learners (time, cultural differences, life way, etc.).

In order to minimise these matters and to adapt our lessons to different kinds of people, situations and contexts, we proposed to work on the modelling and deployment of flexible pedagogical scenarios. Thus it is possible, thanks to EMLs to describe an activity on a higher level and then to concretely transform it, at the runtime, thanks to different sort of treatment, depending on the technical possibilities available in situ. We achieve this by using our COW platform which plays the role of an intermediation platform between teachers and learners. We also take into account the user device, his/her student profile, and particular contextual data given or detected during the interaction. We predominantly tried to offer mobile communications possibilities to our students, using standards frameworks and languages (VoiceXML, WML, XHTML) in the deployment phase. Further work will lead us to improve connections between technical feasibility and pedagogical intentions. We are already able to provide, from a high-level document, multiple final documents, to be used on different channels (even in the same time and in a synergic way) and our next step is to insure that the pedagogical intention is not deteriorated within the different transformations on the learning platform. 


\section{Acknowledgements}

The authors are thankful to the MIAOU and MOSAIQUES programmes (French Nord Pas-de-Calais Region) and the UE funds (FEDER) for providing support for this research.

\section{References}

Ark, W. and Selker, T. (1999) 'A look at human interaction with pervasive computers', IBM Systems Journal, Vol. 38, pp.504-507.

Barré, V., El-Kechaï, H. and Choquet, Ch. (2005) 'Re-engineering of collaborative e-learning systems: evaluation of system, collaboration and acquired knowledge qualities', AIED'05 Workshop: Usage Analysis in Learning Systems, Amsterdam.

Bomsdorf, B. (2005) Adaptation of Learning Spaces: Supporting Ubiquitous Learning in Higher Distance Education, Mobile Computing and Ambient Intelligence: The Challenge of Multimedia, Dagstuhl Seminar Proceedings.

Bourguin, G. and Derycke, A. (2005) 'Systèmes interactifs en co-evolution: reflexions sur les apports de la theorie de l'activite au support des pratiques collectives distribuées', Revue d'Interaction Homme-Machine, AFIHM Europia, Vol. 6, p.29.

Chevrin, V., Derycke, A. and Rouillard, J. (2005) 'Some issues for the modelling of interactive e-services from the customer multi-channel interaction perspectives', EEE 05 International. IEEE International Conference on E-Technology, E-Commerce and E-Service (pp.256-259). Hong Kong: IEEE Press.

Chevrin, V., Derycke, A. and Rouillard, J. (2006) 'Project UBI-learn: an intermediation infrastructure multi-channel access to future LMS', Conference IEEE, AICT/ICIW 2006. 19-24 February, La Guadeloupe, France.

Chevrin, V., Rouillard, J. and Derycke, A. (2005) 'Multi-channel and multi-modal interactions in e-marketing: toward a generic architecture for integration and experimentation', HCI International Conference, Las Vegas, Lawrence Erlbaum editors.

Derycke, A., Rouillard, J., Chevrin, V. and Bayart, Y. (2003) 'When marketing meets HCI: multi-channel customer relationships and multimodality in the personalization perspective', HCI International 2003. Heraklion, Crete, Greece, Vol. 2, pp.626-630, Lawrence Erlbaum Associates.

Dewey, J. (1916) Democracy and Education. New York, NY: MacMillan.

Dey, A., Abowd, G. and Salber, D. (2001) 'A conceptual framework and a toolkit for supporting the rapid prototyping of context-aware applications', Human-Computer Interaction, Vol. 16, pp.97-166.

Dillenbourg, P. (2002) 'Over-scripting CSCL: the risks of blending collaborative learning with instructional design', in P.A. Kirschner (Ed.), Three Worlds of CSCL. Can We Support CSCL (pp.61-91). Heerlen: Open Universiteit Nederland.

Dourish, P. (2001) Where the Action Is: The Foundations of Embodied Interaction. Cambridge: MIT Press.

Dourish, P. (2004) 'What we talk about when we talk about context', Personal and Ubiquitous Computing, Vol. 8, pp.19-30.

Fischer, G. (2001) 'User modeling in human-computer interaction', Journal of User Modelling and User-Adapted Interaction (UMUAI), Vol. 11, pp.65-86.

IMS (2003) 'Global learning consortium', IMS Learning Design Information Model, version 1.0 Final Specification.

Kiczales, G., des Rivières, J. and Bobrow D. (1991) The Art of the Metaobject Protocol. Cambridge, MA: The MIT Press, ISBN: 0-262-61074-4. 
Koper, R. (2001) 'Modeling units of study from a pedagogical perspective: the pedagogical meta-model behind EML', Technical Report, Educational Technology Expertise Centre, Open University of the Netherlands. Available at: http://eml.ou.nl/introduction/docs/pedmetamodel.pdf.

Lin, J., Ho, C., Sadiq, W. and Orlowska, M.E. (2001) 'On workflow enabled e-learning services', in Paper presented in the Proceedings of the IEEE International Conference on Advance Learning Technologies, ICALT2001, 6-8 August, Madison, USA.

MDA (2006) 'OMG model driven architecture'. Available at: http://www.omg.org/mda last visited June.

Ogata, H., Yin, C. and Yano, Y. (2004) 'Context-aware support for learning japanese polite expressions', in Paper presented in the Proceedings of the IEEE International Workshop on Wireless and Mobile Technologies in Education, WMTE'04.

OMG (2006) 'Object management group'. Available at: http://www.omg.org last visited June.

Pernin, J-Ph. (2006) 'Normes et standards pour la conception, la production et l'exploitation des EIAH', chapter 9 in M. Grandbastien and J-M. Labat (Eds), Environnements informatiques pour l'apprentissage humain, Hermes, ISBN 2-7462-1171-8.

Peter, Y. and Vantroys, T. (2005) 'Platform support for pedagogical scenarios', Revue Educational Technology and Society, Vol. 8, pp.122-137 (International Forum of Educational Technology and Society - ISSN 1176-3647).

Rouillard, J. (2004) VoiceXML. Le langage d'accès à Internet par téléphone, aux éditions Vuibert, ISBN: 271174826X, p.197, Paris.

Rust, R. and Kannan, P.K. (2003) 'E-service: a new paradigm for business in the electronic environment', Communication of the ACM, Vol. 46, pp.37-42.

Thomas, S. (2005) 'Pervasive, persuasive e-learning: modeling the pervasive learning space', in Paper presented in the Proceedings of the Third IEEE International Conference on Pervasive Computing and Communications Workshops (PERCOMW'05), pp.332-336.

Vantroys, T. (2003) 'Du langage métier au langage technique, une plate-forme flexible d'exécution de scénarios pédagogiques', PhD Thesis. France: University of Sciences and Technologies of Lille.

VoiceXML (2006) 'Forum'. Available at: http://www.voicexml.org last visited June.

WAP (2006) 'Forum'. Available at: http://www.wapforum.org last visited June.

Weiser, M. (1991) 'The computer for the 21st century', Scientific American, Vol. 265, pp.94-104.

Workflow Management Coalition (2002) 'Workflow process definition interface - xml process definition language', WfMC-TC-1025, version 1.0. Available at: http://www.wfmc.org/ standards/docs/TC-1025_10_xpdl_102502.pdf. 